\title{
LHomme
}

L'HOMME Revue française d'anthropologie

218 | 2016

Varia

\section{L'anthropologie ou les promesses du crépuscule}

\author{
Nicolas Adell
}

\section{OpenEdition}

\section{Journals}

Édition électronique

URL : http://journals.openedition.org/lhomme/28927

DOI : 10.4000//homme.28927

ISSN : 1953-8103

\section{Éditeur}

Éditions de l'EHESS

\section{Édition imprimée}

Date de publication : 20 mai 2016

Pagination : 67-83

ISSN : 0439-4216

\section{Référence électronique}

Nicolas Adell, «L'anthropologie ou les promesses du crépuscule », L'Homme [En ligne], 218 | 2016, mis en ligne le 19 mai 2018, consulté le 19 avril 2019. URL : http://journals.openedition.org/

Ihomme/28927 ; DOl : 10.4000/lhomme.28927

(c) École des hautes études en sciences sociales 


\title{
L'anthropologie ou les promesses du crépuscule
}

\author{
Nicolas Adell
}

\section{L'}

ÉCRITURE DE Daniel Fabre sait envelopper son lecteur d'un subtil mélange de bienveillance et de cruauté ${ }^{1}$. Bienveillance du guide qui accompagne son élève le plus ignorant en le prévenant des dangers et des vertiges (de la surinterprétation, de l'anachronisme, du comparatisme sans rivage, etc.) ; cruauté du compagnon qui n'éclaire qu’à peine le sentier sur lequel on s'engage, généralement par un accès assez insolite, et qui contraint à le suivre de près jusqu'à ce qu'imperceptiblement ce qui peinait à être vraiment une piste ne devienne, sous nos pieds, un chemin assuré au bout duquel un horizon est garanti.

La fréquentation de ses textes m'a fait de plus en plus me lancer dans la lecture avec une grande confiance, qui n'effaçait pas la petite cruauté nécessaire à l'effet que doit produire la "révélation " finale, où les pièces du puzzle, jusque-là indépendamment tournées et retournées jusqu’à trouver leur sens adéquat, sont assemblées d'un coup et font surgir la figure recherchée, c'est-à-dire la clé explicative de leur concomitance, de leur voisinage ou de toute autre forme de proximité.

Cet usage régulier des textes de Daniel Fabre m'a également rendu plus disponible au repérage des mécanismes intellectuels les plus couramment utilisés par l'auteur, à sa rhétorique, mais aussi, progressivement, à certaines de ses obsessions qui se sont affirmées, me semble-t-il, dans les dernières années. Je n'entends pas par là des thèmes récurrents, ni des objets intellectuels, et encore moins des terrains privilégiés. Il s'agit plutôt d'une attention constamment portée sur certains types de phénomènes plutôt que sur d'autres, d'une attirance pour des processus dont les manifestations sociales peuvent être très variées, mais qui sont en quelque sorte apparentés.

1. Au sens tout à fait positif du terme tel que décrit par Clément Rosset (1988). 


\section{Apocalypse}

68

Cela ne m'est apparu en toute clarté que très récemment, à l'occasion du tournage des séquences destinées à produire le dix-huitième épisode de la série L'Ethnologie en héritage, consacrée à la présentation synthétique de la vie et de l'œuvre de quelques-uns des anthropologues de langue française les plus importants ${ }^{2}$. Pour préparer au mieux les six à huit heures de tournage qui étaient programmées, Daniel, qui mavait sollicité pour mener l'entretien avec Alain Morel (ministère de la Culture et de la Communication), m'avait transmis des textes qui lui paraissaient signi-

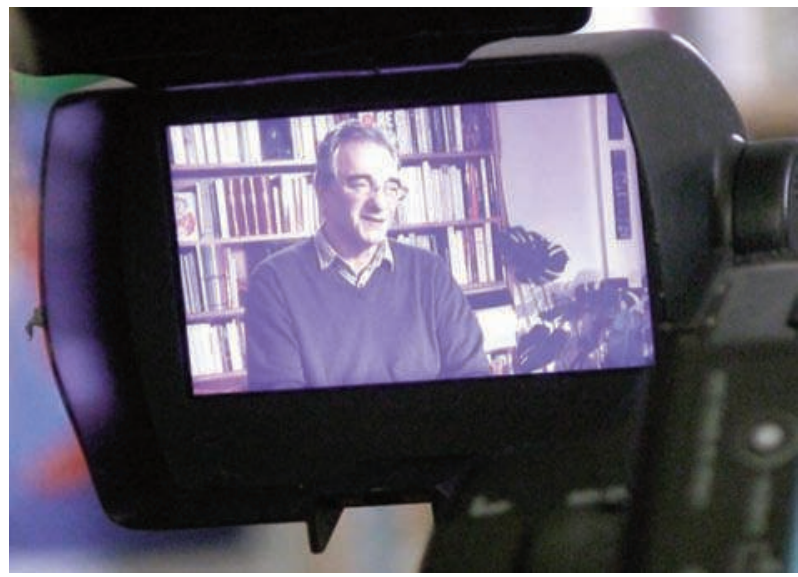

"Dans le viseur" - Daniel Fabre lors du tournage du 18 e épisode de la série L’Ethnologie en héritage 8 décembre 2015 (cl. Ann Gourmelen) ficatifs. Outre ceux d'intention et à contenu clairement autobiographique (Fabre 2010a; Catalini 2012; Fabre 2013a; Wendling 2013), il tenait à ce que j'ai en main le panel le plus révélateur et le plus synthétique de ces travaux, panel retranché de ce qu'il pensait que j'avais déjà médité en raison de mes propres recherches sur l'initiation masculine chez les compagnons ou sur le patrimoine culturel immatériel. Une quinzaine de tirés-à-part au bout du compte, échelonnés entre son article sur "La folie de Pierre Rivière" de 1991 (un texte qui évoque un passage à l'âge d'homme manqué et propose ainsi une autre lecture du dossier révélé par Michel Foucault) et celui, autobiographique encore, clôturant une récente livraison de L'Homme consacrée à la chanson (Fabre 1991a, 2015).

Dans la plupart de ces textes, comme en beaucoup d'autres comme j'ai pu le constater par la suite, un motif revient: celui de la disparition. Présent dès les premiers travaux de Daniel Fabre, ce motif a pris dans les derniers une place de plus en plus centrale, qu'atteste un lexique récurrent évoquant les "fins du monde », les crépuscules et, surtout, les apocalypses. Je ne vois guère de hasard au fait que l'un de ses derniers chantiers,

2. La collection d'entretiens filmés L'Ethnologie en héritage, réalisés par Gilles Le Mao, regroupe à ce jour 17 épisodes (produits par la Huit production et le ministère de la Culture et de la Communication, format $180 \mathrm{~min}$, double DvD), depuis Isac Chiva (2006) jusqu’à Emmanuel Terray (2016). 
conduit avec Giordana Charuty et Marcello Massenzio, ait consisté en une entreprise de traduction du grand livre inachevé d'Ernesto De Martino, La Fine del mondo. Contributo all'analisi delle apocalissi culturali (1977) ${ }^{3}$. Il se dessine là, à mon avis, quelque chose qui serait le noyau d'un " mythe personnel » au sens de l'ancienne critique littéraire (Béguin 1945) et de la psychanalyse (Lacan 1978 [1953]), et qui pourrait fournir une clé de compréhension et d'unification - comme Daniel Fabre aimait dernièrement à la dénicher chez ses «maîtres » (René Nelli, Claude Lévi-Strauss, Pierre Bourdieu, Arnold Van Gennep) ${ }^{4}$ comme chez des savants tombés dans un oubli partiel (Francisque Michel) ou total (Georges Hérelle) ${ }^{5}-$ d'une œuvre buissonnante, portée par la fascination des sentiments apocalyptiques. De celle-ci émergent les contours d'une démarche intellectuelle spécifique - l'anthropologie de l'histoire -, d'une conception singulière du creuset de la discipline - l'anthropologie comme science des mondes finissants - et d'une attention éduquée à l'analyse de certaines situations privilégiées lues comme des moments apocalyptiques par excellence, c'est-à-dire des lieux de dévoilement des fondements de l'expérience historique des sociétés contemporaines, fondements qui étaient pour lui les ressorts mêmes de la condition culturelle de l'homme moderne. Exposons brièvement cette démarche et cette conception, ainsi que le type de situations observées dont elles découlaient.

\section{Une démarche: l'anthropologie de la culture comme anthropologie de l'histoire (à soi)}

Dans un texte récent, Daniel Fabre (2013b) a discuté l'idée assez singulière, et en tous les cas assez distincte de celle des ethnologues français ou anglo-saxons des années 1950 et 1960, qu'Ernesto De Martino se faisait de l'« ethnologie » et en laquelle, avec quelques aménagements, il se reconnaissait lui-même. Ce programme d' "analyse ethnologique", qui est exposé en toute clarté dans La Fine del mondo, relève entièrement des sciences historiques: "L'etnologia è la scienza storica dell'etnos",

3. L'ouvrage devrait paraître à l'automne 2016, dans la collection "Translations" des éditions de l'EHESS.

4. Sur René Nelli, on pourra lire Daniel Fabre \& Jean-Pierre Piniès (1987, 2011 : 7-13); et sur Claude Lévi-Strauss, Daniel Fabre (2012). Les travaux sur Pierre Bourdieu ont fait l'objet de plusieurs séances de son séminaire à l'EHESS, tandis qu'il achevait, au moment de son décès, un texte important sur Arnold Van Gennep, pour ouvrir le livre issu du grand colloque qu'il avait organisé en octobre 2011, avec Christine Laurière, sur l'auteur de Manuel du folklore français.

5. Cf. Daniel Fabre (2010b) pour Francisque Michel. Sur Georges Hérelle, le texte, achevé en 2015, paraîtra aux éditions du CTHS dans un volume que je dirige avec Jérôme Lamy, intitulé : Ce que la science fait à la vie. 
écrit Ernesto De Martino (1977 : 400). En ce sens, il se dissocie très explicitement des perspectives de Claude Lévi-Strauss dont la matrice est profondément naturaliste ${ }^{6}$. Les faits de culture auraient la propriété des faits de nature, "hormis ", écrit Daniel Fabre, "la reproductibilité expérimentale» (Fabre 2013b: 151).

Ces perspectives contrastées impliquaient une confrontation dans les démarches et les ambitions, mais également dans les modalités de production des faits scientifiques ethnologiques. À l'élaboration de la distance objectivante sur le modèle des sciences de la nature par la méditation des sciences du langage chez Claude Lévi-Strauss, Ernesto De Martino oppose un "ethnocentrisme critique", qui intègre à la construction de la problématisation ethnologique la question de la posture de l'observateur et de la nécessaire réflexivité dont il doit faire preuve. Autrement dit, le problème n'est pas l'ethnocentrisme en soi, c'est l'« ethnocentrisme dogmatique» (De Martino 1977: 396) dont l'anthropologie de Lévi-Strauss n'est pas dépourvue, selon De Martino.

Conscient des enjeux de ces propositions et des perspectives qu'elles ouvraient, ainsi que de l'apport incontestable de la démarche structuraliste à une élucidation d'une gamme très large de comportements et de savoirs, Daniel Fabre a réussi le tour de force d'appliquer sur la toile de fond d'une ethnologie demartinienne les outils intellectuels de l'approche lévi-straussienne. Cela signifiait ne pas congédier la question du sens - comme il pouvait reprocher à certains collègues de le faire - mais en déplacer le traitement dans la détermination du champ (dynamique) où le fait (l'objet, l'événement, le parcours, le texte, etc.) signifie. Or, l'une des clés d'une telle identification tenait, pour Daniel Fabre, dans la prise en compte d'un élément fondamental souligné par Ernesto De Martino : le rôle instaurateur de la "rencontre ethnographique " comme révélateur des deux cultures en présence et de leur rapport différentiel qui définit une certaine historicité pratique.

On comprend dès lors que la caractéristique essentielle de l'ethnologie demartinienne relevée par Daniel Fabre est d'être une "anthropologie de l'histoire» (Fabre 2013b : 153-158). Mais l'expression est à bien des égards trompeuse et a prêté à nombre de malentendus, qui ont pu être productifs, mais pas toujours, et nécessitent en tous les cas des éclaircissements. "L'anthropologie de l'histoire n'est ni de l'histoire anthropologisée ni de l'anthropologie historique ni ce qu'on pourrait appeler de la macrohistoire ", écrit-il (Ibid.: 153). Et il reprend une expression de Norbert 
Elias pour la qualifier, expression qui résonnait vivement avec son souci de combiner Ernesto De Martino et Claude Lévi-Strauss : c'est "une histoire structurée" (Ibid.). Histoire structurée dont les principes de structuration ne sont pas donnés à l'avance, mais s'élaborent de proche en proche par l'examen systématique des contacts, des rencontres, de la série des conjonctions et des disjonctions qui donnent forme à l'espace et au temps adaptés à l'objet de recherche. C'était le fondement même d'une anthropologie du symbolique à laquelle, dans le domaine européen, il a apporté une contribution majeure.

Cette anthropologie de l'histoire - en laquelle il réunissait des œuvres aussi contrastées que celles de De Martino, Gauchet, Foucault, Lefort, Dumont, Ariès - consistait donc fondamentalement en une ethnologie de la rencontre, souvent en situation d'inégalité vive, rencontre qui avait la vertu d'un embrayeur et d'un révélateur de la conscience historique (et culturelle) d'un individu, d'un groupe, d'un monde. C'est un aspect que Daniel Fabre a élevé au rang de principe essentiel, généralisant ce qu'Ernesto De Martino avait réservé à quelques situations exceptionnelles témoignant de grandes «apocalypses culturelles » explicitées et théorisées par les acteurs eux-mêmes. Dès lors, il fallait rechercher dans toute rencontre le potentiel apocalyptique qu'elle pouvait receler - et qui pouvait transparaitre dans la tristesse banale de l'informateur de ne pas parvenir à faire accéder son interlocuteur à la totalité de son monde et dans l'incompréhension mutuelle - et qui, seul, contenait l'énergie nécessaire à l'instauration de l'enquête ethnologique.

D'où l'attention que Daniel Fabre prêtait aux réflexivités autochtones, aux "auto-ethnographies" qui lui paraissaient les ethnologies les plus fondamentales, en ce sens qu'elles se déclenchaient justement sous l'effet d'un sentiment d'apocalypse (présente ou imminente) et ne subissaient pas l'effet supplémentaire d'effacement accompagnant le compte rendu d'un tiers au "regard éloigné ». La traduction n'est jamais assez proche, la mémoire jamais assez fidèle, l'expérience jamais assez intense. Les travaux qu'il avait consacrés aux "artistes de la mémoire " (Fabre 2005, 2010c), peintres et écrivains qui re-présentent par le seul pouvoir de l'anamnèse le monde perdu ${ }^{7}$, et qu'il projetait de poursuivre ${ }^{8}$, $s^{\prime}$ inscrivent dans cette dynamique et cette conviction.

7. Il avait d'ailleurs fait récemment l'acquisition de plusieurs œuvres de l'un de ces peintres, le Tunisien Jaber al Mahjoub (né en 1938), aujourd'hui " pris " par l'art brut.

8. Notamment dans le cadre d'un projet de programme ANR, «Désir de pérennité : anthropologie comparée des dispositifs de "mise en valeur" des biens d'exception", à l'initiative duquel il se trouvait. 
L'anthropologie avait ainsi pour lui, parmi ses tâches spécifiques, à répondre à un petit nombre de questions essentielles : "Comment ce qui est reçu est vécu et pensé en même temps? Comment sommes-nous dans l'histoire? Comment sommes-nous présents à l'histoire?» (Wendling 2013). Tel est, à mon sens, le questionnement qui définit l'historicité pratique. La très haute valeur qu'il accordait à la réflexivité, domaine en lequel comme en beaucoup d'autres il estimait que la littérature avait largement devancé les sciences humaines et sociales, doit se comprendre à la lumière de ces interrogations autour de la présence à soi, au monde et dans le temps, interrogations qui ne pouvaient véritablement être traitées qu'à partir de ratages ou de crises. Ces dernières étaient susceptibles de prendre des dimensions spectaculaires, comme dans le cas de celles étudiées par Ernesto De Martino. Elles pouvaient également être repérées à partir de fissures plus modestes, mais n'en témoignaient pas moins de l'extrême fragilité de l'existence au sein du régime apocalyptique qui définit notre rapport actuel au temps ${ }^{9}$. Pour en rendre compte, il fallait savoir abolir méthodiquement la fameuse distance ethnographique - plus proche de la méthode de Germaine Tillion que de celle de Claude Lévi-Strauss ${ }^{10}$ - sans parvenir à ces états d'empathie fusionnelle qu'il considérait comme impropres à la production du savoir. Là encore, il se retrouvait parfaitement dans la perspective de l' " ethnocentrisme critique » d'Ernesto De Martino, qu'il déployait singulièrement vers une ethnologie $\mathrm{du}$ soi. La dimension autobiographique de plusieurs de ses textes, implicite d'abord puis de plus en plus explicite ${ }^{11}$, combinait ainsi les vertus de l'expérience "suffisamment" vécue - puisqu'il s'agissait de sa vie même - avec les ressorts de l'appareil critique qui devaient donner à cette expérience personnelle les contours de l'espace et du temps qui la rendent signifiante, c'est-à-dire qui en font une expérience partagée avec d'autres que soi ${ }^{12}$.

9. Il me semble que, par des voies différentes, François Hartog (2016: 176-177) témoigne de préoccupations similaires dans la détermination du régime d'historicité qu'il qualifie de "présentisme".

10. Sur ce contraste éthique et méthodologique, cf. Christian Bromberger (2015) et Tzvetan Todorov (2016).

11. Sans être exhaustif, on pourra lire deux exemples de cet "égocentrisme critique" implicite dans Daniel Fabre (1977 - et le commentaire révélateur de Pelen [2001] - et 1996) et deux cas explicites dans Daniel Fabre (2009 - avec un "aveu » essentiel dès la première note; lire ici même le texte d'Agnès Fine qui la cite et la commente, p. 31 - et 2015).

12. Giordana Charuty a fourni la clé de la résonance "personnelle» du problème des «crises de la présence» chez Ernesto De Martino dans un livre récent (2010: 57-59). Une découverte à laquelle Daniel Fabre a été très sensible et qu'il a tâché de mettre en perspective (Fabre \& Massenzio 2013). 


\section{Une conception de la discipline : le savoir des crépuscules}

À l'occasion du tournage de l'épisode de L'Ethnologie en héritage et tandis

que nous évoquions les travaux nombreux qu'il a consacrés à l'histoire de la discipline, Daniel a improvisé ce qui lui semblait être, dès l'origine, la caractéristique distinctive de l'anthropologie par rapport à l'ensemble des sciences voisines. Il constatait que toutes les sciences sociales se sont construites, au XIX ${ }^{\mathrm{e}}$ siècle, le regard tourné vers l'avenir, projetant la société telle qu'elle était vers ce qu'elle pouvait être, lisant le futur des relations sociales, des rapports au temps ou à l'art, dans les signes livrés par l'observation du présent. Les historiens même n'échappaient pas à cette tendance - qui est un phénomène recouvrant mais ne se superposant pas exactement à l'idéologie du progrès -, ménageant des chapitres de "prévision historique", une dimension qui restait suffisamment vive dans les années 1940 pour que Marc Bloch envisage d'en faire le sujet du dernier chapitre de son livre resté inachevé, Apologie pour l'histoire ou Métier d'historien ${ }^{13}$. Or, selon Daniel Fabre, l'anthropologie, à rebours de cette enquête générale sur les commencements, s'est dès le départ appliquée à l'enregistrement des fins. Son milieu d'éclosion est celui des mondes finissants, un milieu qui laisse une marque puissante sur la discipline et qui s'exerce bien au-delà de la nature des objets de recherche. Elle nécessite de ce fait de son praticien un pouvoir d'évocation qui peut se nourrir des mécanismes de la narration historique comme de ceux de la littérature, et soumet ce dernier à la tentation permanente du «sauvetage » (qu'elle partage avec l'archéologie), ce qui en fait la complice "naturelle» des entreprises actuelles de patrimonialisation de la culture. Et ce sont précisément ces moments crépusculaires mais épistémologiquement inauguraux qui ont donné à l'enquête anthropologique ses premiers instruments conceptuels: la notion de "survivance", celle de "culture " (associée à l'idée de transmission, d'héritage, de mémoire, posant tôt le problème de l'authenticité ${ }^{14}$ ), puis de "terrain " et de "participation ", comme si le diagnostic objectif et distancié qui nourrit la prévision, et supposant la solidité relativement désincarnée que demain viendra, devait être remplacé par l'expérience subjective et l'identification qui président à la restitution d'une fragilité essentielle placée sous le signe de l'incertitude.

13. On pourra consulter désormais l'édition critique du texte établie par Étienne Bloch (Bloch 2006). Pour une présentation générale de l'état de ce "problème de la prévision " chez Marc Bloch et au-delà, cf. Peter Schöttler (2005).

14. En référence à un article célèbre d'Edward Sapir (1924). 
La sensibilité de l'anthropologie aux mondes finissants a fait surgir, symptômes de cette attention et causes de son maintien tout à la fois, des êtres d'exception que Daniel Fabre a nommés "individus-monde", formant le creuset du paradigme séminal de la discipline qu'il a qualifié de "paradigme des derniers ${ }^{15}$. Trois figures avaient particulièrement focalisé son intérêt : celle de Dersou Ouzala, le «dernier " des Goldes (ou Hezhen), évoqué dans l'autobiographie du géographe russe Vladimir Arseniev (2007 [1921-1923]) où il relate son expédition dans la province de l'Oussouri en Sibérie extrême-orientale, entre 1902 et 1907 ; celle d'Ishi, le «dernier» des Yahi (Californie), célébré par les anthropologues Alfred Kroeber et Thomas Waterman après qu'ils l'eurent «découvert " dans la prison d'Oroville en $1911^{16}$; et, plus près de nous, celle de Gaston Dominici, le «dernier» paysan, sur laquelle il avait particulièrement travaillé (Fabre 2007a). Chacun manifestait ainsi, à son degré le plus extrême, ce soir des mondes qui fonde l'enquête anthropologique et dont Daniel Fabre avait livré tôt sa conception dans un article important consacré au texte de Carlo Levi, Le Christ sest arrêté à Eboli (Fabre 1990). Cet article devait constituer le chapitre d'ouverture d'un ouvrage qu'il avait intitulé La Bibliothèque des îles, dont nous possédons la plupart des pièces, mais qui sont disséminées dans des revues ou des livres collectifs.

Mais si ce "paradigme des derniers" fonde l'enquête anthropologique, il ne lui est pas réservé. Daniel Fabre était extrêmement sensible à la restitution du plasma intellectuel à partir duquel l'anthropologie avait pris une consistance et dans le voisinage duquel elle se trouvait. Le " paradigme des derniers " en venait dès lors à caractériser une manière de produire un "savoir des différences », qui se distinguait des précédentes approches, marquées selon lui d'abord par l'effet de l'altérité radicale (paradigme de la rencontre de l'Autre, de l'Antiquité au XVIII siècle), puis par celui de la volonté de contrôle et de gestion de l'altérité en général (paradigme $\mathrm{du}$ gouvernement des autres, XVIII ${ }^{\mathrm{e}}$ et $\mathrm{XIX}^{\mathrm{e}}$ siècles). À chacun de ces trois paradigmes correspondaient des instruments propres (le regard descripteur, la statistique classificatrice, l'écoute sensible) et des figures emblématiques (Hérodote, le premier historien-enquêteur connu; Joseph-Marie de Gérando, l'auteur du premier questionnaire anthropologique connu; Bérose, le dernier prêtre de Baal, qui vécut au III siècle av. J.-C. et transmit à ses interlocuteurs grecs ses connaissances des rites et des dieux de la religion babylonienne disparue) ${ }^{17}$.

15. L'article essentiel est Daniel Fabre (2008).

16. L'histoire a été rendue célèbre par Theodora Kroeber qui publia, en 1961, à partir des notes de son époux Alfred, Ishi in Two Worlds (1968 pour la traduction française). 
Or, pour le cerner exactement, il fallait restituer la "préhistoire» du paradigme crépusculaire, dont les traces remontaient, selon Daniel Fabre, à l'Antiquité et dont l'emblématisation par un Bérose (plutôt que par un Dersou ou un Ishi, ou même par un Aphéridon, le «dernier» mazdéen que met en scène Montesquieu dans les Lettres persanes [Fabre 2008: 274-280]) montre l'importance que l'enquête d'épistémologie historique avait pour lui ${ }^{18}$ dans toute élaboration critique du mode de connaissance anthropologique. Il appliquait en ce domaine comme en tous ceux, pourtant très différents, qu'il avait pu aborder, la même règle méthodologique: ne pas borner a priori l'objet par des limites de temps et d'espace. Ce principe de décloisonnement avait rendu nécessaire la critique de l'enfermement dans le "contexte ", qu'il avait tôt adressée, avec Dominique Blanc, en utilisant l'exemple en marche, en l'occurrence l'analyse de l'affaire et des récits des méfaits d'un brigand audois (Blanc \& Fabre 2015 [1982]), plutôt que la lecture accusatrice des travaux des collègues ${ }^{19}$. Critique qu'il continuait d'adresser de cette manière avec ses pièces de La Bibliothèque des îles, parmi lesquelles celle, inédite à ce jour mais dont il avait donné un aperçu à Toulouse à l'occasion d'une journée d'études et d'hommages à sa collègue et amie Agnès Fine en octobre 2015, sur Les Immémoriaux de Victor Segalen et Le Guépard de Giuseppe Tomasi di Lampedusa. Romans apocalyptiques s'il en est, dont il s'était appliqué à démontrer l'identité de noyau, c'est-à-dire celle des soirs définitifs qui annoncent les aurores ethnologiques.

L'effet de décloisonnement radical est d'ailleurs poussé jusque dans ses plus extrêmes retranchements, puisque ces apocalypses séminales ne produisent pas seulement chez les intellectuels l'insistance d'une enquête ou d'un récit de type ethnologique. Elles exercent sur la plupart des individus, pris malgré eux dans le paradigme, une «force» qui, si elle ne déclenche pas de l'ethnologie proprement dite, provoque une attention

17. Pour une idée de la vision panoramique qu'il avait de ces façons distinctes de produire un "savoir des différences", on pourra se reporter à Daniel Fabre (2007b).

18. Cette vaste enquête d'épistémologie historique de l'anthropologie devait aboutir à la production de quatre volumes collectifs, qui n'auraient pas véritablement respecté un principe chronologique (contraire à sa démarche d'anthropologie de l'histoire). L'architecture générale était pensée, mais seul le premier, consacré au "moment romantique", a pu voir le jour (Fabre \& Privat 2010); le deuxième, sur le "moment réaliste » et concocté avec la complicité de Marie Scarpa, était en voie d'achèvement (il ne manquait que son propre texte) ; le troisième, sur le "moment colonial ", était mené avec Christine Laurière et André Mary, et peut s'appuyer sur le séminaire que les trois collègues ont dirigé ensemble en 2012-2013 et 2013-2014 à l'EHESS; enfin, le quatrième volume, qui n'était alors que projeté et dont il aurait probablement assuré la direction avec son ami Jean Jamin, devait être consacré au "moment des Lumières".

19. Cette approche a fait l'objet d'un débat vif, précisément autour des risques et des dimensions de ce desserrement contextuel, entre Jean-Claude Chamboredon et Jean Jamin. Les textes de ce débat ont été joints dans la réédition de l'ouvrage (Blanc \& Fabre 2015 [1982] : 238-271). 
aiguisée à son avatar dans l'ordre de l'action publique de la culture : le patrimoine culturel. Or, l'émergence du concept tel que nous le mobilisons aujourd'hui était fondamentalement liée, pour Daniel Fabre, à cette apocalypse que fut la Seconde Guerre mondiale. Destruction radicale mais créatrice d'un rapport neuf au temps et à la culture, rapport qui définit la patrimonialité. Dès lors, toute situation portant la marque apocalyptique, quel qu'en soit le degré (destruction de monuments, d'emblèmes, de paysages ; disparition de pratiques), déclenchera l'identification patrimoniale ${ }^{20}$.

\section{Des situations privilégiées: apocalypses, grandes et "petites"}

Peut-il y avoir de "petites» apocalypses? La disparition d'un monde, qui est en même temps le moment où ses mécanismes les plus profonds se révèlent, peut sans doute prendre des formes très diverses, du meurtre de masse à la lente agonie d'un dernier locuteur - motif largement mythique du thème de la disparition des langues ${ }^{21}$-, de la destruction d'une ville à la "restauration » sauvage d'un monument, qui sont incomparables quant à leurs effets généraux et leurs conséquences matérielles, et, surtout, humaines. Mais la "grandeur» n'est pas, pour l'analyste, tout entière dans l'effet; elle est aussi dans la nature du processus apocalyptique et des déclenchements affectifs et culturels qu'il provoque dans un mode privilégié, le récit. En ce sens, il n'est pas de "petites » apocalypses. Mieux, ce sont celles dont les conséquences sont en apparence les moins dramatiques, les plus anecdotiques ou les moins bruyantes, et qui ne sont pas perturbées par l'autre "grandeur ", celle des effets, qui seraient susceptibles de nous renseigner le mieux sur l'embrayeur apocalyptique.

Et il me semble que ce sont justement à ces disparitions de faible écho que Daniel Fabre a prêté la plus grande attention. Cela a pu faire penser à certains que son anthropologie esquivait les questions sociales les plus brûlantes et que sa saisie du "contemporain » se limitait à l'accompagnement paisible des acteurs de la patrimonialisation ${ }^{22}$. On a manqué,

20. Sur la présentation des travaux de Daniel Fabre portant sur les questions patrimoniales, ainsi que sa posture de chercheur impliqué en ce domaine, je renvoie au texte de Chiara Bortolotto et Sylvie Sagnes dans cette livraison (pp. 45-56).

21. Je remercie Jean-François Courouau d'avoir attiré mon attention sur cet aspect.

22. Je partage entièrement le sentiment qu'exprime Claudie Voisenat dans les dernières lignes de son introduction au volume Le Tournant patrimonial. Mutations contemporaines des métiers du patrimoine (à paraitre aux Éditions de la MSH), qu'elle rédigeait au moment où survînt la mort du maître et ami : "Daniel Fabre n'était ni un défenseur du patrimoine, ni l'ethnologue des survivances d'un passé imaginé». Je remercie très chaleureusement l'auteure de m'avoir communiqué son texte. 
dans ces diagnostics lapidaires, l'essentiel de la démarche et des principes élaborés, à savoir que la valeur d'une voix et de son déchirement ne saurait se mesurer dans l'impact de son retentissement, et qu'un monde qui disparaît dans le mutisme le plus complet n'en constitue pas moins une disparition. Une «émotion patrimoniale " (Fabre, ed. 2013) renseigne sans doute sur les représentations de l'identité collective et sur l'idée que l'on se fait du patrimoine. Mais elle est d'abord une émotion, et la description de son déclenchement, de ses manifestations et des pratiques qu'elle engendre participe pleinement d'une anthropologie contemporaine des affects.

D'ailleurs, les plus fortes de ces «émotions» sont celles qui, au mouvement physiologique, intérieur et individuel, ajoutent l'expression d'un mouvement social, extérieur et collectif renouant avec le sens premier du terme (Ibid. : 36-37). Dans les dernières années, Daniel Fabre avait de ce point de vue concentré ses enquêtes sur les indices d'une sorte d' "expression obligatoire des sentiments" (Mauss 1921) devant les menaces pesant sur les biens culturels et dont la valeur est attestée par le caractère spontané des mouvements que ces menaces déclenchent. Face aux transformations projetées du parvis révélé de l'église SaintVincent de Carcassonne, face au projet de dérestauration de la basilique Saint-Sernin de Toulouse, face aux conflits d'interprétation (religieuse, culturelle, spirituelle, ésotérique, etc.) des espaces «sacrés» de Vézelay ${ }^{23}$, la mise en place de mobilisations spontanées, de mouvements coordonnés, et toute la gamme des expressions de l'émotion collective le passionnaient, non seulement par la nature des déclencheurs (le patrimoine, entre autres), mais aussi par les formes sociales neuves engendrées, la créativité des manifestations, les innovations coopératives et les voies nouvelles de l'action collective. Il était convaincu, dans un esprit très proche de celui de Georges Bataille qui lui servait explicitement de référence, de la puissance créatrice (dans les registres tout à la fois social et intellectuel) des destructions d'objets, des disparitions de pratiques, des «apocalypses culturelles » de petite envergure ${ }^{24}$.

Il avait également observé ce phénomène dans les émotions, les réactions vives voire les soulèvements qui marquaient l'effacement progressif des manières traditionnelles de sanctionner les comportements sociaux

23. Pour un aperçu du caractère inaugural des dossiers Saint-Sernin et Saint-Vincent, lire Daniel Fabre \& Christian Hottin (2011). L'épaisse et complexe affaire Vézelay a fait l'objet d'une enquête pluriannuelle, conduite sous la direction de Daniel Fabre et Claudie Voisenat (Fabre 2014a; Voisenat 2016). Les deux complices avaient prévu de réunir leurs textes pour en faire un livre; souhaitons que ce projet aboutisse.

24. Une première formulation explicite de cette conviction se trouve dans Daniel Fabre (2002a). Et pour un examen de ce phénomène dans le cadre "replié " des fêtes qui en sont comme le laboratoire, cf. Daniel Fabre (2014b). 
" anormaux" (charivari, asouade, etc.) ou de ponctuer la vie collective et dont le temps de carnaval lui semblait un noyau essentiel. L'on ne disparaît pas toujours dans la tristesse du soir, mais aussi dans des résistances joyeuses qui maintiennent la coutume collective contre l'enflement de la vie privée (Fabre 1999 [1986]) et, pour ce faire, s'adaptent, se transforment, innovent $^{25}$. L'ethnographie des formes de la fin, qu'il avait dès les années 1970 pensée comme un programme de recherche, avait justifié en partie la construction du Centre d'anthropologie des sociétés rurales à Toulouse avec son " pays " Jean Guilaine ${ }^{26}$. Il se plaisait à en rappeler la rhétorique : "Tandis que Jean et les archéologues s'occupaient des commencements du monde paysan au Néolithique, nous, les anthropologues, nous chargions de l'enregistrement de sa disparition" (comm. pers.).

En réalité, c'est le changement de point de vue, le passage de l'observation des manifestations collectives de résistance à celle des ratages individuels qui suscite l'effet "tristesse du soir", en détournant quelque peu l'expression de Roland Barthes (2002 : 176), que Daniel Fabre a pu lire dans la défense pathétique de Gaston Dominici (Fabre 2007a), ou dans la "folie» de Pierre Rivière qui est, en fait, "conscience, aiguë jusqu’à la douleur, de l'ordre perdu des coutumes"(Fabre 1991a: 122). De ces solitudes de "derniers", un individu se dégageait, qui était sans folie, angoisse ou résignation, et présentait au contraire la vivacité, la résilience dirait-on peut-être aujourd'hui, de ces mouvements collectifs de résistance, mais qui seraient sans autre action que celle des " actes de parole " propres au conteur qui a le pouvoir de faire resurgir des mondes, à l'instar des " artistes de la mémoire»: Pierre Pous (né en 1929), conteur exceptionnel du pays de Sault, fut la rencontre ethnographique décisive. Son humour, ses traits de génie, son accent, sa joie de dire les contes ouvraient le problème anthropologique de la disparition du monde dont il persistait à assurer la présence: "Comment sommes-nous dans l'histoire? Comment sommes-nous présents à l'histoire? " sont des questions qui ont sans doute pris forme dans cette rencontre et au cours des très nombreuses heures que Daniel Fabre a passées à écouter et à enregistrer le conteur du hameau de Munès (Aude), dès la fin des années 1960. Surtout, Pierre Pous témoignait d'une réflexivité exceptionnelle sur son monde en train de disparaître, qui le chargeait tout à la fois des habits du dernier homme et du premier auto-anthropologue qu'il ait pu fréquenter. L'anthropologie

25. Lire, par exemple, le travail qu'il avait mené avec Bernard Traimond sur le charivari en Gascogne (Fabre \& Traimond 1981).

26. Pour cette histoire institutionnelle, je renvoie au texte de Jean Guilaine associé à cet hommage (pp. 13-22). Et pour un témoignage sur l'esprit de l'« école de Toulouse » insufflé par Daniel Fabre, à celui d'Agnès Fine (pp. 23-34). 
de la culture comme anthropologie de l'histoire que concevait Daniel Fabre lui doit beaucoup, au point qu'il envisageait la rédaction d'un volume qui aurait complété et actualisé sa thèse sur le conte occitan de tradition orale (Fabre \& Lacroix 1974), dont le sujet aurait été le conteur Pous :

"Si j'ai le temps, un jour, je voudrais rééditer ma thèse avec un troisième volume qui lui serait consacré en tant que conteur. J'aimerais le lui offrir en espérant qu'il soit toujours là » (in Wendling 2013).

Il ne l'écrira pas, et l'on ne saura donc pas ce qu'il comptait y révéler. L'on peut en revanche supposer l'argument général qui lui aurait servi de fil conducteur, en parcourant ce qu'il avait pu dire d'une autre rencontre, essentielle et séminale, celle de l'intellectuel et polygraphe René Nelli (1906-1982) qui eut lieu le 5 mai 1963 (Fabre 2011: 69) ${ }^{27}$. Il présentait, sous de notables différences dont le rapport à l'écrit est la principale, les mêmes caractères encyclopédiques, un art similaire du conter, "cette capacité à faire revivre ce qui n'existe plus » (in Wendling 2013), en quoi réside le pouvoir du poète. Le dernier conteur et le poète: deux figures $\mathrm{du}$ " double » ${ }^{28}$, deux figures annonciatrices et " aimantes " (au sens affectif et magnétique) de l'anthropologue dans la conception que s'en faisait Daniel Fabre, et dont il avait décrit les affinités électives à partir du contexte irlandais au tournant du XXe siècle (Fabre 2002b).

Une même conscience historique et culturelle, une intense réflexivité et une capacité spécifique d'en rendre compte unissent ces scrutateurs des crépuscules que sont le conteur, le poète et l'anthropologue. Pourtant Daniel Fabre accordait aux uns et aux autres des qualités différentes: au conteur, la capacité d'actualisation de la culture; à l'anthropologue, le pouvoir d'en décrypter patiemment les processus de transformation; au poète, celui d'en révéler les cohérences profondes. Les programmes intellectuels des deux derniers avaient, selon Daniel Fabre, une grande proximité, que la fréquentation de René Nelli, poète, philosophe, historien et ethnologue, n'avait cessé de confirmer. Mais il était convaincu que le poète gardait toujours une longueur d'avance en raison, d'une part, d'une certaine hauteur de vue qu'il avait continué de mesurer tout particulièrement dans le compagnonnage de René Char et d'André du Bouchet, et d'autre part, d'une légitimité à la fulgurance que l'appareil

27. Je renvoie au texte de Jean Guilaine qui souligne l'importance de René Nelli dans l'orientation de Daniel Fabre (pp. 14-15).

28. Pour Pierre Pous comme « double ", je renvoie aux indications livrées dans le superbe entretien accordé à Thierry Wendling en 2013 (Wendling 2013). Et pour René Nelli, on lira l'explicite et polysémique "Face au double» (Fabre 2011), puisqu'il y est également question du rapport de René Nelli au poète paralysé, Joë Bousquet (1897-1950). 
scientifique interdit: "La science est trop lente", résumait Arthur Rimbaud dans Une saison en enfer. Et c'est à Rimbaud justement que Daniel Fabre avait accordé la paternité du repérage de cette association ancienne, qui l'avait longtemps obsédé à partir d'un conte entendu en gardant les vaches avec Pierre Pous, entre le langage des oiseaux, celui de l'amour et la conquête de la virilité ${ }^{29}$. Une révélation crépusculaire des Illuminations dit "à quelle apocalypse prélude le silence cosmique des oiseaux" (Fabre 2014d: 429, mes italiques). Laissons au poète les derniers mots :

«Les sentiers sont âpres. Les monticules se couvrent de genêts. L'air est immobile. Que les oiseaux et les sources sont loin! Ce ne peut être que la fin du monde, en avançant» (Rimbaud 2009: 292).

Université Toulouse-Jean Jaurès Laboratoire interdisciplinaire, Solidarités, Sociétés, Territoires (LISST) Centre d'anthropologie sociale, Toulouse nicolasadell@yahoo.fr

29. L'œuvre-maîtresse, Le Roi des oiseaux, ou l'Invisible initiation, n'a jamais paru, annoncée depuis longtemps par quelques articles (Fabre 1986, 1991a et b) et, surtout, par des séminaires donnés dans le temps de sa période toulousaine. Ses plus proches collègues et amis ont pu cependant en lire l'économie générale et quelques chapitres qu'il ne cessait de remanier, indice d'une œuvre-vie. Le dernier livre qu'il a publié de son vivant, sur l'invention de l'art préhistorique (Fabre 2014c), en porte encore la marque (Ibid.: 126), comme il l'explicite dans le cadre d'un dialogue noué avec la romancière Danielle Mémoire (en ligne : http ://www.fondation-entreprise-ricard.com/ Conferences/view/374-daniel-fabre-danielle-memoire). Pour une analyse de cet ouvrage, qui souligne la manière dont il unifie différents aspects des travaux de Daniel Fabre, depuis ses enquêtes sur les "dénicheurs d'oiseaux" jusqu'à celles portant sur les "failles de l'être savant ", on pourra se reporter à l'excellente note de lecture d'André Mary (2016). 
Arseniev, Vladimir

2007 [1921-1923] Dersou Ouzala.

Trad. du russe par Pierre P. Wolkonsky.

Éd. par Michel Jan. Paris, Payot \& Rivages

("Petite bibliothèque Payot» 624).

\section{Barthes, Roland}

2002 Comment vivre ensemble: simulations romanesques de quelques espaces quotidiens. Cours et séminaires au Collège de France (1976-1977). Éd. par Claude Coste.

Paris, Le Seuil-ImEC ( Traces écrites»).

\section{Béguin, Albert}

1945 Gérard de Nerval. Paris, José Corti.

Blanc, Dominique \& Daniel Fabre

2015 [1982] Le Brigand de Cavanac. Le fait divers, le roman, l'histoire.

Lagrasse, Verdier ("Poche»).

Bloch, Marc

2006 L'Histoire, la guerre, la Résistance. Éd. par Annette Becker et Étienne Bloch. Paris, Gallimard ("Quarto»).

\section{Bromberger, Christian}

2015 «La méthode Tillion: enquêter, comprendre, intervenir ", in Germaine Tillion, Les Combats d'une ethnologue. Entretiens avec Frédéric Mitterrand. Éd. par Christian Bromberger et Tzvetan Todorov. Paris, Éd. de l'EHESS-INA («Audiographie»12) : 7-30.

\section{Catalini, Alessio}

2012 «France-Italie: un parcours d'anthropologue. Intervista a Daniel Fabre», L'Uomo 1-2 : 175-192.

\section{Charuty, Giordana}

2010 Ernesto De Martino. Les vies antérieures d'un anthropologue. Marseille, Parenthèse-Éd. de la MMSH (" Parcours méditerranéens. Série Savoirs et savants »).

\section{De Martino, Ernesto}

1977 La Fine del mondo. Contributo all'analisi delle apocalissi culturali.

Éd. par Clara Gallini. Torino, Einaudi.

Fabre, Daniel

1977 La Fête en Languedoc. Regards sur le carnaval aujourd'hui. Photographies de Charles Camberoque. Toulouse, Privat.

1986 «La voie des oiseaux : sur quelques récits d'apprentissage ", L'Homme 99 : 7-40.

1990 "Carlo Levi au pays du temps", L'Homme 114 : 50-74.

1991a "La folie de Pierre Rivière ", Le Débat 66 : 96-109.

$1991 b$ "Une enfance de roi", Ethnologie française 21 (4) : 392-414. 1996 " "Faire la jeunesse" au village", in Giovanni Levi \& Jean-Claude Schmitt, eds, Histoire des jeunes en Occident, 2. L'époque contemporaine. Paris, Le Seuil ("L'Univers historique ») : 51-83.

1999 [1986] «Familles : le privé contre la coutume ", in Philippe Ariès \& Georges Duby, eds, Histoire de la vie privée, 3. De la Renaissance aux Lumières. Paris, Le Seuil («Points. Histoire» 262) : 527-564. 2002a «Catastrofe, scoperta, intervento o il monumento come evento ", in Andreina Ricci, ed., Archeologia e urbanistica. Firenze, All'Insegna del Giglio : 19-28.

2002b «Le berceau de la langue:

John Millington Synge aux îles d'Aran ", L'Homme 163 : 19-50.

2005 "Peindre la mémoire ", L'Homme 175-176 : 251-276.

2007a «Le dernier paysan : à propos de l'affaire Dominici ", Lengas. Revue de sociolinguistique 61 : 117-135.

2007b «Les savoirs des différences: histoire et sciences des mœurs en Europe (XVIII ${ }^{\mathrm{e}}-\mathrm{XX}$ e siècles) ", in Karine Chemla (avec la collab. de Mireille Delbraccio), ed., Histoire des savoirs, 2003-2007. Recueil de synthèses. Paris, CNRS-MESR : 65-68. 
2008 «Chinoiserie des Lumières: variations sur l'individu-monde", L'Homme 185-186 : 269-300.

2009 « Fondu au noir », L'Homme 191: 27-36.

2010a "Préface : un récit des origines", in Jean Guilaine, Un désir d'histoire.

L'enfance d'un archéologue. Carcassonne, Garae Hésiode : 7-21.

2010b «Francisque Michel :

la fin de la polygraphie romantique", in Daniel Fabre \& Jean-Marie Privat, eds, Savoirs romantiques... : 103-128.

2010c "L'enfer de la mémoire", L'Homme 195-196 : 125-162.

2011 "Face au double ", in Daniel Fabre \& Jean-Pierre Piniès, eds, 31 vies et revies de René Nelli... : 69-74.

2012 « D'Isaac Strauss à Claude Lévi-Strauss : le judaïsme comme culture", in Philippe Descola, ed., Claude Lévi-Strauss, un parcours dans le siècle. Paris, Odile Jacob ("Travaux du Collège de France») : 267-294. 2013a "Allocution de M. Daniel Fabre", in Une épée de bronze pour Jean Guilaine. Arles, Actes Sud-Errance : 30-34.

2013 b «Ernesto De Martino, La Fin $d u$ monde et l'anthropologie de l'histoire ", Archives de sciences sociales des religions 161 : 147-162.

2014a Conquérir Vézelay. Territoires, culture, spiritualités. Rapport au Département du pilotage de recherche et de la politique scientifique. Paris, Ministère de la Culture et de la Communication.

2014b «Construire, détruire: le temps syncopé des objets de la fête ", in Agnès Barruol, Yves Cranga \& Hélène Palouzié, eds, Regards sur le patrimoine des fêtes et des spectacles. Arles, Actes Sud : 20-37.

2014c Bataille à Lascaux. Comment l'art préhistorique apparut aux enfants. Paris, L'Échoppe.

2014d "Langage des oiseaux et idée de la poésie ", in Jean-François Courouau, François Pic \& Claire Torreilles, eds, "Amb un fil d'amistat". Mélanges offerts à Philippe Gardy... Toulouse, Centre d'étude de littérature occitane : 415-430.
2015 "Rock des villes et rock des champs", L'Homme 215-216 : 233-250.

2016 «Inversion et dislocation : les vies savantes de Georges Hérelle ", in Nicolas Adell \& Jérôme Lamy, eds, Ce que la science fait à la vie. Paris, Éd. du CTHS [à paraître].

Fabre, Daniel, ed.

2013 Émotions patrimoniales. Paris, Éd. de la MsH ( Cahiers d'ethnologie de la France " 27).

\section{Fabre, Daniel \& Christian Hottin}

2011 «Entretien entre Daniel Fabre et Christian Hottin : le patrimoine saisi par l'événement ", Livraisons d'histoire de l'architecture 22 : 51-58.

Fabre, Daniel \& Jacques Lacroix 1974 La Tradition orale du conte occitan. Les Pyrénées audoises. Paris, Presses universitaires de France, 2 vol.

Fabre, Daniel \& Marcello Massenzio 2013 «Vies parallèles: Ferdinand de Saussure avec Ernesto De Martino ", L'Homme 205 : 137-152.

Fabre, Daniel \& Jean-Pierre Piniès, eds 1987 René Nelli et les "Cahiers du Sud". Carcassonne, Garae Hésiode.

201131 vies et revies de René Nelli. Vidas e razos. Carcassonne, Garae Hésiode.

Fabre, Daniel \& Jean-Marie Privat, eds 2010 Savoirs romantiques. Une naissance de l'ethnologie. Nancy, Presses universitaires de Nancy ("EthnocritiqueS ).

\section{Fabre, Daniel \& Bernard Traimond}

1981 "Le charivari gascon contemporain : un enjeu politique ", in Jacques Le Goff \& Jean-Claude Schmitt, eds, Le Charivari. Paris, Éd. de l'EHESS -Mouton (“Civilisations et sociétés " 67) : 23-32.

\section{Hartog, François}

2016 "Vers une nouvelle condition historique ", Le Débat 188 : 169-180. 
Kroeber, Theodora

1961 Ishi in Two Worlds. A Biography

of the Last Wild Indian in North America.

Berkeley, University of California Press.

1968 Ishi. Testament du dernier Indien sauvage de l'Amérique du Nord. Trad. par Jacques Hess. Paris, Plon ("Terre humaine»).

Lacan, Jacques

1978 [1953] «Le mythe individuel

du névrosé ", Ornicar? 17-18: 290-307.

Mary, André

2016 «Daniel Fabre: à propos de Bataille à Lascaux. Comment l'art préhistorique apparut aux enfants", Ethnologie française 161 : 165-170.

Mauss, Marcel

1921 "L'expression obligatoire des sentiments ", Journal de psychologie 18 : 425-434.

\section{Pelen, Jean-Noël}

2001 «Identité narrative et ethnographie: en amical hommage à Daniel Fabre et Charles Camberoque ", Cahiers de littérature orale 50 : 261-278.

\section{Rimbaud, Arthur}

2009 Euvres complètes.

Éd. par André Guyaux avec la collab. d'Aurélia Cervoni. Paris, Gallimard («Bibliothèque de la Pléiade»).

\section{Rosset, Clément}

1988 Le Principe de cruauté.

Paris, Minuit ("Critique»).

\section{Sapir, Edward}

1924 "Culture, Genuine and Spurious", American Journal of Sociology 29 (4):

401-429.

\section{Schöttler, Peter}

2005 «Marc Bloch, die Lehren der Geschichte und die Möglichkeit historischer Prognosen ", Österreichische Zeitschrift für Geschichtswissenschaften 16 (2) : 104-125.

\section{Todorov, Tzvetan}

2016 «Deux approches des sciences humaines : Lévi-Strauss et Germaine Tillion ", Le Débat 188 : 181-192.

\section{Voisenat, Claudie}

2016 L'Éloge du ressenti. Le patrimoine entre médiations spirituelles et lectures ésotériques. Rapport au Département du pilotage de recherche et de la politique scientifique. Paris, Ministère de la Culture et de la Communication [à paraître].

\section{Wendling, Thierry}

2013 «L'intelligence du conte : entretien avec Daniel Fabre », ethnographiques.org 26 [http ://www.ethnographiques.org/L-intelligence-du-conte-Entretien\#nh4]. 